\title{
Evaluation of s.c. route of immunization by homologous radio attenuated live vaccine in experimental murine model of visceral leishmaniasis
}

\author{
Sanchita Datta $\cdot$ Supriya Khanra $\cdot$ Anindita Chakraborty $\cdot$ \\ Syamal Roy $\cdot$ Madhumita Manna
}

Received: 26 February 2014/ Accepted: 9 August 2014/Published online: 20 September 2014

(C) Indian Society for Parasitology 2014

\begin{abstract}
Our previous studies in BALB/c mice showed substantial protection against the experimental murine visceral leishmaniasis (MVL) when the animals were immunized with $\gamma$-irradiated live Leishmania donovani parasites through intra peritoneal (i.p.) and intra muscular (i.m.) routes respectively. The observations encouraged us to check the prophylactic efficacy of subcutaneous (s.c.) route as it is better alternative for human trial. The mice immunized with two subsequent doses of the radio attenuated homologous vaccine were challenged with virulent $L$. donovani parasites. Seventy-five days post infection, the animals were sacrificed. The extent of protection against the disease was evaluated by assessing the reduction of parasite burden in spleen and liver, the generation of free radicals (NO \& ROS) and release of the cytokines from T-lymphocyte helper 1 (Th 1) and T-lymphocyte helper 2 (Th 2) along with the measurement of the serum immunoglobulins. The reductions in parasitic burden were observed up to 21 and $24 \%$ in spleen and liver of the immunized groups with NO and ROS productions 27 and $34 \%$ respectively. Whereas the increase in IFN gamma releases was between 19 and $34 \%$, the decrease in IL-10 release was not more than $22 \%$. This indicates the failure of the establishment of
\end{abstract}

S. Datta $\cdot$ S. Khanra $\cdot$ M. Manna $(\bowtie)$

Post Graduate Department of Zoology, Barasat Govt. College,

10, K.N.C Road, Kolkata 700124, India

e-mail: madhumita.manna09@gmail.com

A. Chakraborty

UGC-DAE Consortium for Scientific Research, Kolkata Centre,

3/LB-8, Salt Lake, Kolkata 700098, India

S. Roy

Infectious Diseases and Immunology, Indian Institute of

Chemical Biology, 4, Raja S.C. Mullick Road, Kolkata 700032,

India pronounced Th1 ambience which was further corroborated by the observed IgG2a and IgG1 ratio. The present study when compared with our previous observations with i.m. and i.p. routes revealed that s.c. route may not be a good choice for the use of radio attenuated vaccine.

Keywords Visceral leishmaniasis .

Radio attenuated vaccine .

Routes of homologous immunization · NO/ROS .

Th1/Th2 cytokines

\section{Introduction}

Leishmaniases are tropical vector borne disease conditions caused by the unicellular kinetoplastid protozoa, Leishmania sp. The diseases are prevalent in six continents and endemic in 88 countries and annually about two million of new cases are reported worldwide with almost 350 million people are at risk (Desjeux 2004). On the bases of the clinical manifestations and tissue tropisms of the parasites, there are three major forms of the disease: cutaneous, visceral, and mucosal. World health organization (WHO) gives high priority to visceral leishmaniasis (VL) or Kalaazar (KA) and is fatal if not treated (World Health Organization 2006). High risk of HIV co infection in the endemic zones makes the control strategy for VL vulnerable (WHO/UNAIDS 1998). Uses of the current available drugs such as Antimonials, Amphotericin B, Pentamidine etc. are far from satisfaction due to the emergence of resistance and the cost and side effects respectively (Croft et al. 2006). The observation that recovery from VL confers protection from re infection gives strong impetus for vaccine development to combat the disease. The cell mediated protective immunity against Leishmaniasis is largely attributed to an 
Interleukin-12 (IL-12) driven T helper-1 (Th1) response and Interferon-gamma (IFN- $\gamma)$ production resulting in the macrophage activation which ultimately kills the parasites. On the other hand, an IL-10 driven T helper-2 (Th2) response has been recorded in disease progression in murine model of infection (Haldar et al. 2009). Elevated level of IL-10 has also been reported in patients with active VL and they have shown increased expression of IL-10 mRNA in their bone marrow and lymph nodes with higher levels of IL-10 in sera (Saha et al. 2007; Gautam et al. 2011). Extensive vaccine studies against experimental animal models of the disease have encompassed the uses of live/ killed parasites to nonliving proteins as vaccine candidates (Afrin et al. 2000; Bhowmick and Ali 2009; Bhaumik et al. 2009; Mukhopadhyay et al. 2000). DNA subunit vaccines tested so far have not shown much hope as they did not impart long-term immunity (Mukhopadhyay et al. 1999) and the killed vaccines have not performed satisfactorily in the field trials (Basu et al. 2005). The live-attenuated vaccine, on contrary, provides an attractive alternative as it mimics the natural infection for some time and could display a plethora of antigens to the antigen presenting cells in the host. This may lead to similar immune response as that would be seen in case of infection with virulent parasites (Handman 2001). Factors related to the efficacy, production easiness and cost effectiveness of live attenuated leishmanial vaccines have also made them attractive immunizing candidate against VL.

In the present study and also in our previous study, we have taken radio-attenuated Leishmania donovani parasites as our vaccinating agent against the disease caused by virulent $L$. donovani. Gamma irradiations at three different doses [50 Gray (Gy), $100 \mathrm{~Gy}$ and 150 Gy respectively] were used for the attenuation of the virulent $L$. donovani parasites. We got encouraging results using this homologous vaccine candidate in both our prophylactic and therapeutic studies using i.p. and i.m. routes (Datta et al. 2010; Datta et al. 2011; Datta et al. 2012) respectively. The present study entails our previous experiments and intends to understand the immunological response pattern in s.c. route in murine model as it is considered as one of the preferred routes for human trial.

\section{Materials and methods}

Chemicals and reagents

Penicillin, Streptomycin, HEPES, Sodium bicarbonate, 2-mercaptoethanol (2-ME), sulfanilamide, N-(1-naphthyl) ethylene diamine hydrochloride, M199 medium, RPMI1640,Giemsa stain, TMB, $\mathrm{H}_{2}$ DCFDA $\left(2^{\prime}\right.$, $7^{\prime}$-dichlorodihydrofluorescein diacetate) were purchased from Sigma-Aldrich.
Fetal Bovine Serum (FBS) was purchased from Gibco, USA. Enzyme Linked Immunosorbent Assay (ELISA) kit was purchased from BD-Bioscience, USA and Mouse IgG assay kit was obtained from Zymed, USA.

Animals, parasites and infection

BALB/c mice of 4-6 week old with almost equal weight and same sex, reared in Institute facilities, were used for experimental purposes and all animal experimentations were performed at Indian Institute of Chemical Biology, Kolkata, India following the National Regulatory Guidelines issued by Committee for the Purpose of Control and Supervision of Experiments on Animals (CPCSEA), Ministry of Environment and Forest, Govt. of India. L. donovani strain Ag83 (MHOM/IN/1983/AG83) (Manna et al. 2005) originally obtained from confirmed Indian Kala-azar patient, were maintained in Golden hamsters (Mukhopadhyay and Madhubala 1994). Promastigotes obtained after transforming the amastigotes from infected animal spleen were cultured in medium M 199 supplemented with $10 \%$ FBS along with $100 \mathrm{U} / \mathrm{ml}$ Penicillin and $100 \mu \mathrm{g} / \mathrm{ml}$ Streptomycin and maintained at $22{ }^{\circ} \mathrm{C}$. Mice were injected with $5 \times 10^{6}$ stationary phase promastigotes suspended in Phosphate buffered Saline (PBS) through intra cardiac route (Mukhopadhyay et al. 1999).

Immunization with attenuated parasites

Leishmania donovani parasites were attenuated by Gamma-irradiator machine (UGC-DAE, Kolkata Centre, India) at three different doses, 50 Gray (Gy), $100 \mathrm{~Gy}$, 150 Gy respectively. The description of the animal groups (five animals in each group) for the present study was as follows: Group 1, normal control animals without any infection and immunization; Group 2, infected control animals with infection and without immunization while Group 3, Group 4 and Group 5 were animals received two doses of immunization through s.c. route with Leishmania donovani parasites $\left(5 \times 10^{6}\right.$ parasites/animal $)$ attenuated at $50 \mathrm{~Gy}, 100 \mathrm{~Gy}$ and $150 \mathrm{~Gy}$ doses of gamma radiation respectively. The immunization was repeated for three immunized groups in 15-day interval. Fifteen days after the last immunization, the mice were infected with same number of virulent parasites intra cardiac. The mice finally were sacrificed 75 days post infection in order to check different experimental parameters.

Determination of parasite burden in spleen and liver

Splenic and hepatic parasite burden of the BALB/c mice of different groups were determined by microscopic evaluation of Giemsa stained tissue imprints. Parasite per spleen or liver was counted as-no. of amastigotes $\times 2 \times 10^{5} \times$ 
weight of liver or spleen (in $\mathrm{mg}$ ) per 1,000 nucleated cells where $2 \times 10^{5}$ is a derived constant (Stauber 1963).

Preparation of soluble parasite antigen

Leishmanial lysates from washed promastigotes $(10 \% / \mathrm{ml})$ were prepared by several cycles (minimum six) of freezing $\left(-70{ }^{\circ} \mathrm{C}\right)$ and thawing $\left(37^{\circ} \mathrm{C}\right)$ followed by five minutes of incubation on ice (Mukhopadhyay et al. 1999). Partially lysed promastigotes were then disrupted by sonication thrice with pulse of $30 \mathrm{~s}$ each (Soniprep 150; MSE, UK) and centrifuged at $10,000 \mathrm{rpm}$ for $30 \mathrm{~min}$ at $4{ }^{\circ} \mathrm{C}$. The supernatant containing soluble antigen was collected and the protein concentration was determined by Bradford Protein Assay method (Bio-Rad). Prepared antigen was stored at $-20{ }^{\circ} \mathrm{C}$ until further use.

Quantification of nitric oxide (NO)

The NO production was evaluated by measuring the accumulation of nitrite in the culture medium of the splenocytes (Green et al. 1982). Splenocytes $\left(2 \times 10^{6} \%\right.$ animal) from different groups of experimental mice were incubated in 24-well tissue culture plate with $5 \mu \mathrm{g} / \mathrm{ml}$ SLA in $10 \%$ FBS containing RPMI-1640 medium for $48 \mathrm{~h}$ at $37{ }^{\circ} \mathrm{C}$ in $5 \% \mathrm{CO}_{2}$ incubator. After $48 \mathrm{~h}$ of incubation, the supernatant was collected. The mixture of Griess Reagent and culture supernatant at 1:1 ratio was incubated in 96-well ELISA plate for $20 \mathrm{~min}$ at room temperature in dark. The OD was determined at $550 \mathrm{~nm}$ by spectrophotometer (Smart spec 3000; Bio-Rad, USA).

\section{Measurement of reactive oxygen species}

To measure the reactive oxygen species (ROS) which include superoxide, hydrogen peroxide and other reactive oxygen intermediates, the cell permeable, nonpolar, $\mathrm{H}_{2} \mathrm{O}_{2}$ sensitive probe $2^{\prime}, 7^{\prime}$ - Di Chlorofluorescein Di Acetate was used (Haldar et al. 2009). In brief, $2 \times 10^{6}$ splenocytes from different groups of BALB/c mice were stimulated with $5 \mu \mathrm{g} / \mathrm{ml} \mathrm{SLA}$ for $24 \mathrm{~h}$ by keeping at $37{ }^{\circ} \mathrm{C}$ in $5 \%$ $\mathrm{CO}_{2}$ incubator. After $24 \mathrm{~h}$ of incubation, the splenocytes were washed and resuspended in PBS and incubated with $2 \mu \mathrm{g} / \mathrm{ml} \mathrm{H}_{2}$ DCFDA at room temperature for $20 \mathrm{~min}$ in the dark. Relative fluorescence was measured (LS50B spectrophotometer, Perkin-Elmer, USA) at an excitation wavelength of $510 \mathrm{~nm}$ and emission wavelength of $525 \mathrm{~nm}$ and the measurement was expressed as arbitrary units.

Assay of Th1 and Th2 cytokines

Single cell suspension of splenocytes from all experimental groups of $\mathrm{BALB} / \mathrm{c}$ were prepared after Ficoll density gradient centrifugation (Datta et al. 2010) and then suspended in $10 \%$ FBS containing RPMI-1640 medium. The splenocytes were plated in 96-well tissue culture plates in presence of $5 \mu \mathrm{g} / \mathrm{ml} \mathrm{SLA}$ in triplicate at $2 \times 10^{5}$ cells/well and incubated at $37{ }^{\circ} \mathrm{C}$ in $5 \% \mathrm{CO}_{2}$ incubator for $18-24 \mathrm{~h}$. After incubation, the supernatants were collected to perform cytokine assay by ELISA as per manufacturer's instructions (BD Sciences, USA).

Measurement of antileishmanial antibody responses

Blood was collected from the experimental BALB/c heart pre-sectioning. From the blood, serum was collected and analyzed to determine SLA specific antibody titer. 96-well ELISA plates were coated with SLA $(2 \mu \mathrm{g} / \mathrm{ml})$ in PBS at $4{ }^{\circ} \mathrm{C}$. IgG1 and IgG2a present in the collected sera were measured with the ZYMED Antimouse IgG assay kits (Bhaumik et al. 2009).

\section{Statistical analysis}

The statistical significance of differences between groups was determined by the unpaired two-tailed Student's $t$ test. Statistical significance was defined as $\mathrm{P}$ value of $<0.05$, and results were expressed as averages and standard deviations of triplicate measurement.

\section{Results}

Reduction in parasite burden in the $\mathrm{BALB} / \mathrm{c}$ mice immunized through s.c. route

We evaluated the outcome of challenge infection in mice following vaccination with radio attenuated parasites through subcutaneous route in three immunized groups (designated as 50 Gy group, 100 Gy group and 150 Gy group respectively, Fig. 1a and b) and compared with infected control (Infected Group, Fig. 1a and b). It was seen that Leishman Donovan Unit (LDU) in the spleens of 100 Gy Group and 150 Gy Group were 15 and $21 \%$ less than that in the spleen of the infected animals group (Infected Group, Fig. 1a). Animals of 50 Gy Group did not show any reduction in spleen LDU with respect to the Infected control group indicating parasites treated with 50 Gy dose of gamma irradiation, was not properly attenuated. This observation corroborated our earlier finding with i.p. data (Datta et al. 2011). In case of liver (Fig. 1b), the reductions of LDU in the $50 \mathrm{~Gy}, 100 \mathrm{~Gy}$ and $150 \mathrm{~Gy}$ Groups of animals were 16, 22 and $24 \%$ respectively compared to that of the Infected Group. 


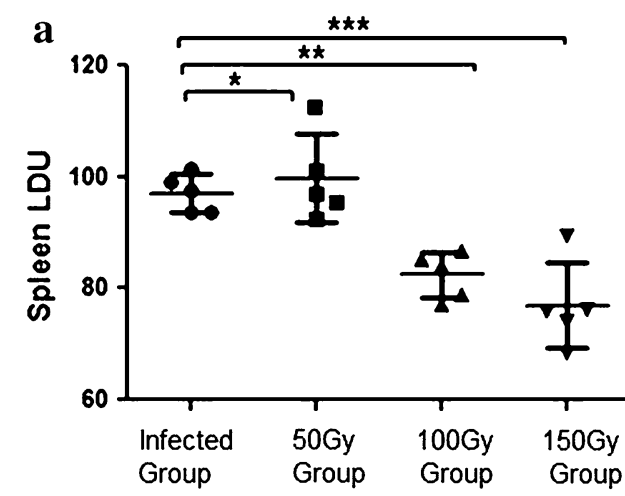

Fig. 1 The parasite burden (expressed as the Leishman Donovan Unit, LDU) of spleen a and liver $\mathbf{b}$ of the BALB/c mice groups are shown. 4-6 weeks old mice were immunized by sub-cutaneous route with gamma irradiated Leishmania donovani parasites, the dosage of radiation being 50 Gray (50 Gy Group), 100 Gray (100 Gy Group) and 150 Gray (150 Gy Group) respectively with one repeat of immunization in fifteen-day interval. 15 days after the last immunization, the mice were infected with virulent $L$. donovani parasites. An

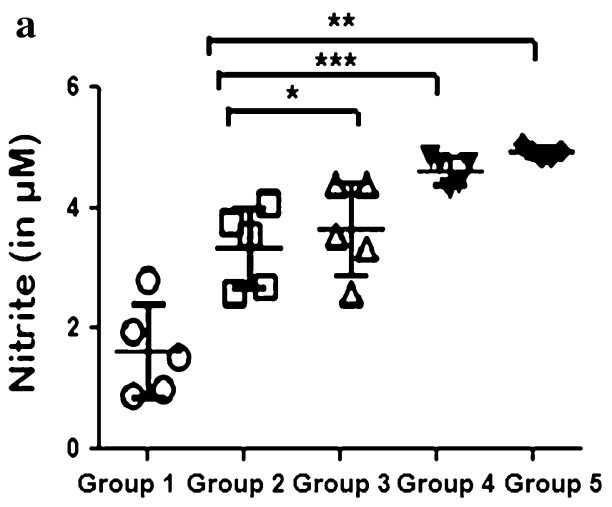

Fig. 2 Nitric oxide levels a and reactive oxygen species levels $\mathbf{b}$ of normal control (Group 1), infected control (Group 2), $50 \mathrm{~Gy}$ immunized mice (Group 3) 100 Gy immunized animals (Group 4) and 150 Gy immunized animals (Group 5) are shown. Experiments

Level of NO and ROS in experimental animal groups

In order to check the success or failure of s.c. route of immunization for radio attenuated parasites as vaccine candidate, we checked the level of free radicals in the experimental system by measuring NO production (Fig. 2a) and reactive oxygen species generations (Fig. 2b) respectively. To study the free radical levels, we stimulated the splenocytes of the experimental animals ex vivo with $5 \mu \mathrm{g} / \mathrm{ml}$ SLA (Mukhopadhyay et al. 1999) and measured the nitrite level. NO-mediated macrophage effector mechanism is critical in the control of $L$. donovani replication in the mouse (Murray and Nathan 1999). The level of nitrite increased by about $7 \%$ in Group 3 (Fig. 2a), $21 \%$ in Group 4 (Fig. 2a) and $27 \%$ in Group 5 (Fig. 2a) respectively compared to that found in the infected animal group,

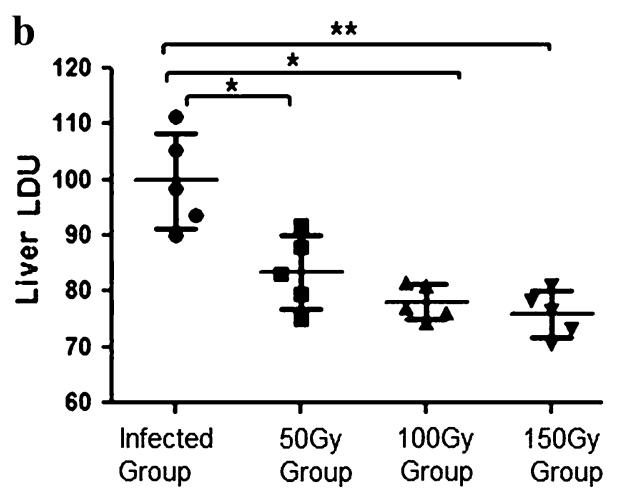

infected control group without immunization (Infected Group) and a normal control group without any infection or immunization were kept along with above groups. The LDU in the three immunized groups were shown along with the infected control group. Data represents mean $\pm \mathrm{SD}$ of five animals per group and are representative of three independent experiments; unpaired two-tailed Student's $t$ test was performed. $P<0.05$ was considered significant. $* P<0.05, * * P<0.01, * * * P<0.001$

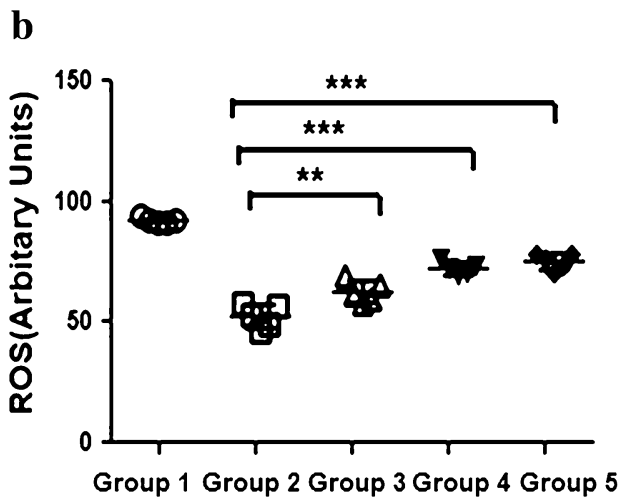

were repeated thrice with five animals per group and one representative data set was shown as mean $\pm \mathrm{SD}$ values; unpaired two-tailed Student's $t$ test was performed. $P<0.05$ was considered significant. $* P<0.05, * * P<0.01, * * * P<0.001$

Group 2 (Fig. 2a). The other arm of parasite killing by macrophage is by the Reactive Oxygen Species. The ROS levels were higher by 11,21 and $34 \%$ times in Group 3(Fig. 2b), Group 4 (Fig. 2b) and Group 5 (Fig. 2b) respectively than that of infected animal group (Fig. 2b).

Th1/Th2 cytokine responses in mice immunized through s.c. route

We set up our experiment to check the level of two most important cytokines IFN- $\gamma$ (Fig. 3a) and IL-10 (Fig. 3b) in our experimental model since it is well accepted that IFN $-\gamma$ is the most important cytokine related to the host recovery from disease (Nathan et al. 1984), while IL-10 is global immunosuppressive Th 2 type of cytokines (Nylen and Sacks 2007) that has profound influence on the disease 


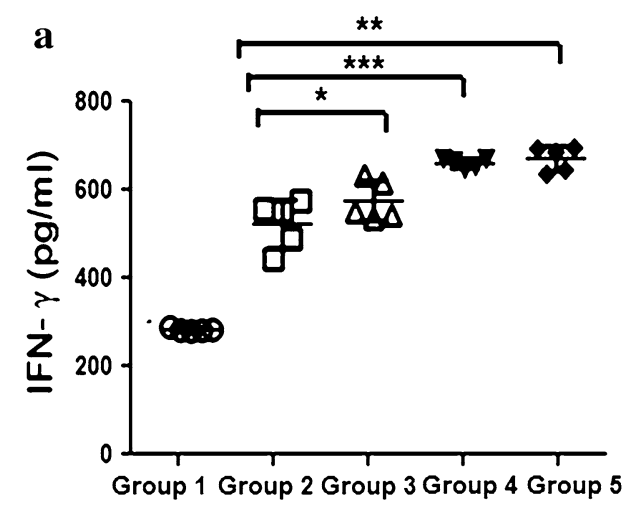

Fig. 3 The release of two different cytokines IFN- $\gamma$ a and IL-10 b of experimental animal groups: Group 1, normal mice without any infection or immunization; Group 2, animals infected with virulent $L$. donovani parasites; Group 3, animals immunized with attenuated parasites irradiated at 50 Gray (Gy) of dose of Gamma radiation; Group 4, animals immunized with attenuated parasites irradiated at

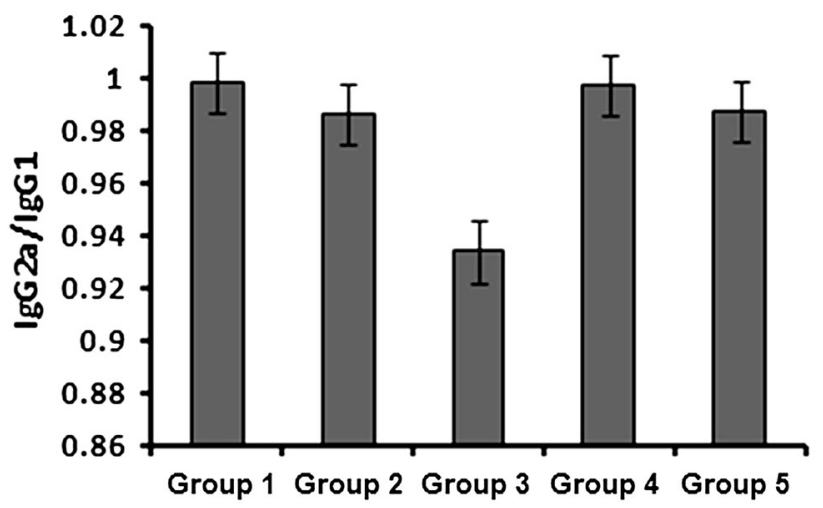

Fig. 4 Humoral immunoglobulin ratio of $\operatorname{IgG} 2 \mathrm{a} / \mathrm{IgG} 1$ in the five animal groups: Group 1, normal mice without any infection or immunization; Group 2, animals infected with virulent $L$. donovani parasites; Group 3, animals immunized with attenuated parasites irradiated at 50 Gray (Gy) of dose of Gamma radiation; Group 4, animals immunized with attenuated parasites irradiated at $100 \mathrm{~Gy}$ of dose and Group 5, animals immunized with attenuated parasites irradiated at $150 \mathrm{~Gy}$ of dose respectively. Data represents mean \pm SD of five animals per group and are representative of three independent experiments; Student's $t$ test was performed and $P<0.05$ was considered significant

exacerbation. The levels of IFN- $\gamma$ were found 19, 25 and $34 \%$ higher in Group 3 (Fig. 3a), Group 4 (Fig. 3a) and Group 5 (Fig. 3a) animals than that found in the infected animals Group (Group 2) (lane 2, Fig. 3a). The level of IL10 was decreased by less than $10 \%$ in Group 3 (lane 3, Fig. 3b) while 15 and $22 \%$ decreases were noticed in Group 4 (Fig. 4b) and Group 5 (Fig. 3b) compared to the IL-10 secretion found in infected control group (Fig. 3b).

Level of humoral responses in s.c. immunized mice

Protection in immunized mice is correlated with a specific pattern of immunoglobulin production as they are surrogate

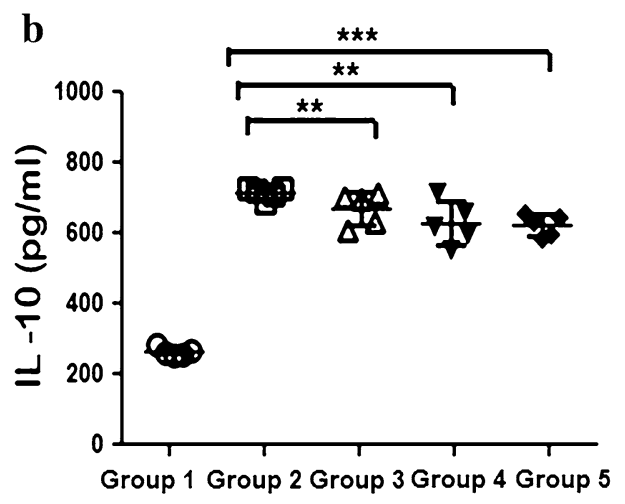

100 Gy of dose and Group 5, animals immunized with attenuated parasites irradiated at $150 \mathrm{~Gy}$ of dose respectively. Data represents mean $\pm \mathrm{SD}$ of five animals per group and are representative of three independent experiments; unpaired two-tailed Student's t test was performed and $P<0.05$ was considered significant. $* P<0.05$, $* * P<0.01, * * * P<0.001$

markers for $\mathrm{CD}^{+}$T-cell populations. The experimental mouse sera were assayed for anti-leishmanial Immunoglobulin $\mathrm{G}$ (Ig G) responses. The ratio of $\mathrm{IgG} 2 \mathrm{a} / \mathrm{IgG} 1$ isotypes (Fig. 4) was checked as these are the markers of Th1 and Th2 $\mathrm{CD}^{+}{ }^{+} \mathrm{T}$-cell differentiation (McMahon-Pratt and Alexander 2004). The blood sera were collected sacrificing the animals and the anti leishmanial IgG1 and IgG2a levels were checked separately at different dilutions. We considered the $10^{-3}$ dilution for both the antibodies and measured the ratio of the two isotypes at this dilution. It was observed that in Group 2, Group 3, Group 4 and Group 5 animals, the ratio $(\mathrm{IgG} 2 \mathrm{a} / \mathrm{IgG} 1)$ were $0.998,0.934$, 0.997 and 0.987 respectively (Fig. 4).

\section{Discussion}

The selection of the proper route of inoculation for a particular vaccine varies from one vaccine candidate to other. The influences of different routes on evoking immune response and the subsequent impact on challenge infection are still unknown. The route of immunization with live Leishmania parasites seems to control the immune response and outcome of the disease (Baldwin et al. 2003; Belkaid et al. 2000; Constant et al. 2000; Menon and Bretscher 1998; Nabors and Farrell 1994). For experimental purposes, intra peritoneal immunization is preferred as pooling of adequate samples are required for cell immunity studies and it results in higher homogeneity in infection (Rolão et al. 2004). To target the human trial for any vaccine candidate, all possible routes should be taken into serious consideration as the immune response thus elicited, may vary from one antigen to other. It was reported earlier that intra dermal immunization is more efficient as it requires less volume (five folds) of vaccine 
solution than that in both intramuscular (i.m.) and s.c. routes (Méndez et al. 2002). For Leishmania DNA vaccine, i.m route of vaccination is the preferred route (Bhowmick and Ali 2009). Vaccination with radio-attenuated parasites has been reported earlier in cutaneous leishmaniasis (Liew et al. 1985; Rivier et al.1993) and recently by our group in VL (Datta et al. 2010; Datta et al. 2011; Datta et al. 2012). We dealt with the attenuated live L.donovani parasites as homologous vaccine candidate for experimental murine visceral leishmaniasis (MVL) for the protection and therapy study using i.p. and i.m. routes of immunization. In our previous study, immune responses of the vaccinated $\mathrm{BALB} / \mathrm{c}$ mice demonstrated that animals immunized through i.m. route showed up to $80 \%$ reduction in LDU in the spleen of immunized animal groups while the reduction of liver LDU were above $90 \%$ in these groups compared to that in the parasitized animals (Datta et al. 2012). $\mathrm{BALB} / \mathrm{c}$ mice immunized through i.p. route have also shown $88 \%$ reduction in spleen LDU and liver LDU respectively (Datta et al. 2010; Datta et al. 2011). In the present experiment for s.c. route, it has been seen that parasite load in spleens of above mentioned groups have been reduced up to $21 \%$ and in case of the reduction in hepatomegaly, the value was $24 \%$. Thus the reduction of parasite burden in the s.c. route immunized groups of animals was much inferior to that observed both in i.m. and i.p. routes. It is already known that free radicals have the central role in parasite clearance in the animals (Murray and Nathan 1999). The reactive oxygen species level in the i.m. immunized animal groups were higher than that in s.c. route while the elevation of nitric oxide level was more pronounced in the i.p. route (five times) than both i.m. (two times) and s.c. routes (less than 1.5 times). The extent of releases of NO and ROS in the s.c. route immunized mice indicated the failure of this route of immunization. Also, analysis of cytokines from splenocytes of the immunized mice revealed no skewing of Th1/Th2 response in the s.c. route immunized animals. In the both of our i.m. and i.p. immunization studies (Datta et al. 2010; Datta et al. 2011; Datta et al. 2012), IFN- $\gamma$ level was found about 3.4-4 times higher in immunized groups compared to the parasitized animals whereas the IL-10 level was reduced by $48 \%$ (i.p. route) to about $60 \%$ (i.m. route) in protected groups. On the other hand, our s.c. route study showed increase of IFN$\gamma$ level by about 1.3 times and decrease in IL-10 release maximally by $22 \%$. The humoral immune response measured as the ratio of $\operatorname{IgG} 2 \mathrm{a} / \mathrm{IgG} 1$ showed almost $1: 1$ ratio depicting the inability of this route of immunization to create stronger Th1 ambience in the immunized groups.

The critical dependence on the route of immunization in obtaining protective immunity is well-known. Kaur et al. (2008) have reported a strong Th1 response in BALB/c mice by inoculation through subcutaneous route with low dose of live, whole parasite antigen. This has been corroborated by Tabbara et al. (2005) and they further reported that vaccination with live parasites through subcutaneous route (s.c.) influences cell types recruited in the secondary site of infection and clears parasites efficiently. On contrary, other workers in the field (Mukhopadhyay et al. 1999; Méndez et al. 2002) experienced that s.c. route did not work well for the experimental MVL. Our present study with the radio-attenuated parasites showed little protection through s.c. route. We have observed that in the immunized groups of animals, there was enhanced IFN- $\gamma$ release along with the increased release of IL-10 and thus no skewing towards Th1 bias has been achieved in the immunized groups. This is corroborated by Das and Ali (2012) who claimed that despite very high IFN- $\gamma$, there were high IL- 4 and IL-10 productions in s.c. immunized animals which might lead to vaccine failure and disease progression in VL. Bhowmick and Ali (2009) showed that supernatants derived from antigen-stimulated spleen cells from i.p. immunized animals had activated macrophages to kill parasites in vitro. This was in contrast to the spleen supernatants from mice immunized by the s.c. route which were not potent macrophage activators. Analysis of total IL-10 production by lesion-derived cells using ELISA also demonstrated significantly higher levels of Ag driven IL-10 production (Tabbara et al. 2005).

There are various reasons for the failure of s.c. route in this protection study. It is known that induction of immune response is macrophage dependent and macrophages are the main cells to cause phagocytosis. In case of immunization through s.c. route, the antigens are exposed mainly to the dendritic cells (DC) and DCs are not much "professional" in phagocytosis compared to the macrophages (Wijburg et al. 1998). In case of s.c. route, the primary encounter DC is the main antigen presenting cell (APC) that activates the resting T-cells and immunization itself will generate its own set of T-reg cells. Thus it is better to select a route where T-reg cells will not be overrepresented (Reckling et al. 2008) The skin contains the highest percentage of natural T-reg cells of the body and they can be expanded by the delivery of antigen under the immunogenic condition (Reckling et al. 2008). Hence it is preferred to select a route for vaccination where the T-reg priming and activation will be minimum. This may not be the case here. The failure of protection by the s.c. route may also be attributed by enhanced pre challenge TGF- $\beta$ production following vaccination (Haldar et al. 2009). In conclusion, the vaccination of $B A L B / c$ mice with the radio-attenuated parasites through the s.c route has less protective immunity against pathogenic $L$. donovani challenge. This can be manifested by the lack of macrophages in the site of vaccination and also by the presence of higher level of T-reg cells at that site but this area of research has not been addressed in the present study. 
Acknowledgments The authors acknowledge the Council for Scientific and Industrial Research (CSIR), India for the fellowship of Sanchita Datta, CSIR Senior Research Fellow since April, 2011. The authors also acknowledge UGC-DAE Consortium for Scientific Research, Kolkata Centre, Kolkata for providing the contingency and fellowship of SD at the initial part of the study before she joined CSIR. The authors are thankful to the Director, Public Instructions, Govt. of West Bengal, India and the Principal, Barasat Govt. College, Kolkata and the Director, Indian Institute of Chemical Biology, Kolkata, India for their kind cooperation.

Conflict of interest None.

\section{References}

Afrin F, Anam K, Ali N (2000) Induction of partial protection against Leishmania donovani by promastigote antigens in negatively charged liposomes. J Parasitol 86:730-735

Baldwin TM, Elso C, Curtis J, Buckingham L, Handman E (2003) The site of Leishmania major infection determines disease severity and immune responses. Infect Immun 71:6830-6834

Basu R, Bhaumik S, Haldar AK, Naskar K, De T, Dana SK et al (2005) Kinetoplastid membrane protein-11 DNA vaccination induces complete protection against both pentavalent antimonial-sensitive and -resistant strains of Leishmania donovani that correlates with inducible nitric oxide synthase activity and IL-4 generation: evidence for mixed Th1- and Th2-like responses in visceral leishmaniasis. J Immunol 174:7160-7171

Belkaid Y, Mendez S, Lira R, Kadambi N, Milon G, Sacks D (2000) A natural model of Leishmania major infection reveals a prolonged "silent" phase of parasite amplification in the skin before the onset of lesion formation and immunity. J Immunol 165:969-977

Bhaumik SK, Naskar K, De T (2009) Complete protection against experimental visceral leishmaniasis with complete soluble antigen from attenuated Leishmania donovani promastigotes involves Th1-immunity and down-regulation of IL-10. Eur J Immunol 39:2146-2160

Bhowmick S, Ali N (2009) Identification of novel Leishmania donovani antigens that help define correlates of vaccine-mediated protection in visceral leishmaniasis. PLoS ONE 4:1-10

Constant SL, Lee SK, Bottomly K (2000) Site of antigen delivery can influence $\mathrm{T}$ cell priming: pulmonary environment promotes preferential Th2-type differentiation. Eur J Immunol 30:840-847

Croft SL, Sundar S, Fairlamb AH (2006) Drug resistance in leishmaniasis. Clin Microbiol Rev 10:111-126

Das A, Ali N (2012) Vaccine prospects of killed but metabolically active Leishmania against visceral leishmaniasis. Expert Rev Vaccin 11:783-785

Datta S, Naskar K, Chakraborty A, Manna M (2010) Immunotherapeutic role of radio attenuated Leishmania parasites in experimental murine leishmaniasis. Medimond International Proceedings (no.M815S7113) XIIth International Congress on Parasitology, Melbourne, Australia

Datta S, Adak R, Chakraborty P, Haldar AK, Bhattacharjee S, Chakraborty A et al (2011) Radio-attenuated leishmanial parasites as immunoprophylactic agent against experimental murine visceral leishmaniasis. Exp Parasitol 130:39-47

Datta S, Manna M, Khanra S, Ghosh M, Bhar R, Chakraborty A et al (2012) Therapeutic immunization with radio-attenuated Leishmania parasites through i.m. route revealed protection against the experimental murine visceral leishmaniasis. Parasitol Res 111:361-369

Desjeux P (2004) Leishmaniasis: current situation and new perspectives. Comp Immunol Microbiol Infect Dis 27:305-318
Gautam S, Kumar R, Maurya R, Nylén S, Ansari N, Rai M et al (2011) IL-10 neutralization promotes parasite clearance in splenic aspirate cells from patients with visceral leishmaniasis. J Infect Dis 204:1134-1137

Green LC, Wagner DA, Glogowski J, Skipper PL, Wishnok JS, Tannenbaum SR (1982) Analysis of nitrate, nitrite and [15N] nitrate in biological fluids. Anal Biochem 26:131-138

Haldar AK, Banerjee S, Naskar K, Kalita D, Islam NS, Roy S (2009) Sub-optimal dose of Sodium Antimony Gluconate (SAG)-diperoxovanadate combination clears organ parasites from BALB/c mice infected with antimony resistant Leishmania donovani by expanding antileishmanial T-cell repertoire and increasing IFNgamma to IL-10 ratio. Exp Parasitol 122:145-154

Handman E (2001) Leishmaniasis: current status of vaccine development. Clin Microbiol Rev 14:229-243

Kaur S, Kaur T, Garg N, Mukherjee S, Raina P, Athokpam V (2008) Effect of dose and route of inoculation on the generation of CD4 + Th1/Th2 type of immune response in murine visceral leishmaniasis. Parasitol Res 103:1413-1419

Liew FY, Hale C, Howard JG (1985) Prophylactic immunization against experimental leishmaniasis. IV. Subcutaneous immunization prevents the induction of protective immunity against fatal Leishmania major infection. The J Immunol 135:2095-2101

Manna M, Majumder HK, Sundar S, Bhaduri AN (2005) The molecular characterization of clinical isolates from Indian Kala-azar patients by MLEE and RAPD-PCR. Med Sci Monit 11:220-227

McMahon-Pratt D, Alexander J (2004) Does the Leishmania major paradigm of pathogenesis and protection hold for New World cutaneous leishmaniases or the visceral disease? Immunol Rev 201:206-224

Méndez S, Belkaid Y, Seder RA, Sacks D (2002) Optimization of DNA vaccination against cutaneous leishmaniasis. Vaccine 20:3702-3708

Menon JN, Bretscher PA (1998) Parasite dose determines the Th1/ Th2 nature of the response to Leishmania major independently of infection route and strain of host or parasite. Eur J Immunol 28:4020-4028

Mukhopadhyay R, Madhubala R (1994) Antileishmanial activity and modification of hepatic xenobiotic metabolizing enzymes in golden hamster by 2(3)-tert-butyl-4-hydroxyanisole following infection with Leishmania donovani. Biochem Pharmacol 47:253-256

Mukhopadhyay S, Sen P, Bhattacharyya S, Majumdar S, Roy S (1999) Immunoprophylaxis and immunotherapy against experimental visceral leishmaniasis. Vaccine 17:291-300

Mukhopadhyay S, Bhattacharyya S, Majhi R, De T, Naskar K, Majumdar S et al (2000) Use of an attenuated leishmanial parasite as an immunoprophylactic and immunotherapeutic agent against murine visceral leishmaniasis. Clin Diagn Lab Immunol 7:233-240

Murray HW, Nathan CF (1999) Macrophage microbicidal mechanisms in vivo: reactive nitrogen versus oxygen intermediates in the killing of intracellular visceral Leishmania donovani. J Exp Med 189:741-746

Nabors GS, Farrell JP (1994) Site-specific immunity to Leishmania major in SWR mice: the site of infection influences susceptibility and expression of the antileishmanial immune response. Infect Immun 62:3655-3662

Nathan CF, Prendergast TJ, Wiebe ME, Stanley ER, Platzer E, Remold HG et al (1984) Activation of human macrophages. Comparison of other cytokines with interferon-gamma. J Exp Med 160:600-605

Nylen S, Sacks D (2007) Interleukin-10 and the pathogenesis of human visceral leishmaniasis. Trends Immunol 28:378-384

Reckling S, Divanovic S, Karp CL, Wojciechowski S, Belkaid Y, Hildeman D (2008) Proapoptotic Bcl-2 family member Bim 
promotes persistent infection and limits protective immunity. Infect Immun 76:1179-1185

Rivier D, Shah R, Bovay P, Mauel J (1993) Vaccine development against cutaneous leishmaniasis: subcutaneous administration of radioattenuated parasites protects CBA mice against virulent Leishmania major challenge. Parasite Immunol 15:75-84

Rolão N, Melo C, Campino L (2004) Influence of the inoculation route in BALB/c mice infected by Leishmania infantum. Acta Trop 90:123-126

Saha S, Mondal S, Ravindran R, Bhowmick S, Modak D, Mallick S et al (2007) IL-10- and TGF-beta-mediated susceptibility in kala-azar and post-kala-azar dermal leishmaniasis: the significance of amphotericin B in the control of Leishmania donovani infection in India. J Immunol 179:5592-5603
Stauber LA (1963) Some aspects of immunity to intracellular protozoan parasites. J Parasitol 49:3-11

Tabbara KS, Peters NC, Afrin F, Mendez S, Bertholet S, Belkaid Y et al (2005) Conditions influencing the efficacy of vaccination with live organisms against Leishmania major infection. Infect Immun 73:4714-4722

WHO/UNAIDS (1998) World Health Organization report on Leishmania and HIV in gridlock report, Geneva 12-25

Wijburg OLC, van den Dobbelsteen GPJM, Vadolas J, Sanders A, Strugnell RA, van Rooijen N (1998) The role of macrophages in the induction and regulation of immunity elicited by exogenous antigens. Eur J Immunol 28:479-487

World Health Organization (2006) Control of leishmaniasis. Report by the Secretariat, World Health Organization, Geneva 